\title{
Presence of Ocratoxin (OTA) in wine produced in Sicily (Italy)
}

\author{
Antonio Vella, Graziella Graci, Francesco Olibrio, Valentina Cumbo, Antonello Cicero, Daniele Di Salvo, \\ Giulia Caracappa \& Vincenzo Ferrantelli
}

Istituto Zooprofilattico Sperimentale della Sicilia “A. Mirri”, Via G. Marinuzzi 3, 90129 Palermo, Italy

\begin{abstract}
S Ocratoxin (OTA) occurs naturally in different foodstuffs, including grapes and their derivatives. OTA is a secondary fungal metabolite produced naturally by Aspergillus and Penicillium species. The purpose of this work was to estimate the occurrence of OTA in wine produced in Sicily to obtain a risk assessment of these substances. A total of 470 wine samples were quantitatively analysed by screening analysis method based on immunoenzymatic assay (ELISA) for ochratoxin. Results confirmed the presence of contamination by OTA in only one sample of red wine "Nero D'Avola” from south-oriental Sicily.
\end{abstract}

KEY WORDS

Ochratoxins; wine; ELISA.

Received 22.11.2019; accepted 10.12.2019; published online 20.12.2019

Proceedings of the 4th International Congress on Biodiversity "Man, Natural Habitats and Euro-Mediterranean Biodiversity", November 17th-19th, 2017 - Malta

\section{INTRODUCTION}

Ocratoxin (OTA) occurs naturally in different foodstuffs, including grapes and their derivatives. The first reports of OTA in wine go back to 1996. Since then, many studies have focused on OTA occurrence in products derived from grapes such as dried vine fruit, wine, grape juice, must and vinegar. Red wines have been reported to be contaminated more frequently than white wines, probably due to the different wine-making methods involved. OTA occurrence seems to be higher in wines from Southern European countries: several studies have shown an increase in the amount of OTA in warmer climates. Given the danger posed by OTA, the European Community has recently established a concentration limit of $2 \mu \mathrm{g} / \mathrm{kg}$ in grape juice, must, wine and dried fruit (Commission Regulation $\mathrm{n}$. $1881 / 2006 /$ EC). OTA is a secondary fungal metabo- lite produced naturally by several Aspergillus and Penicillium species; Aspergillus and Penicillum species able to produce OTA occur in temperate and cold climate areas, respectively. The most important OTA-producing species belong to Aspergillus sections Circumdati and Nigri; however, the presence of OTA in grapes and wine is mainly linked to the contamination in the vineyard by species belonging to the Aspergillus section Nigri, the so-called black aspergilli. The major producer of OTA in grapes is Aspergillus carbonarius, though other species belonging to the Nigri and Circumdati sections have also been found to produce the toxin in different Mediterranean countries, such as Spain, Italy, Portugal, in Australia and South America (see also IARC, 1993; Majerus \& Otteneder, 1996; PfohlLeszkowicz \& Manderville, 2007).

The purpose of this work was to estimate the occurrence of OTA in wine commercialized and pro- 


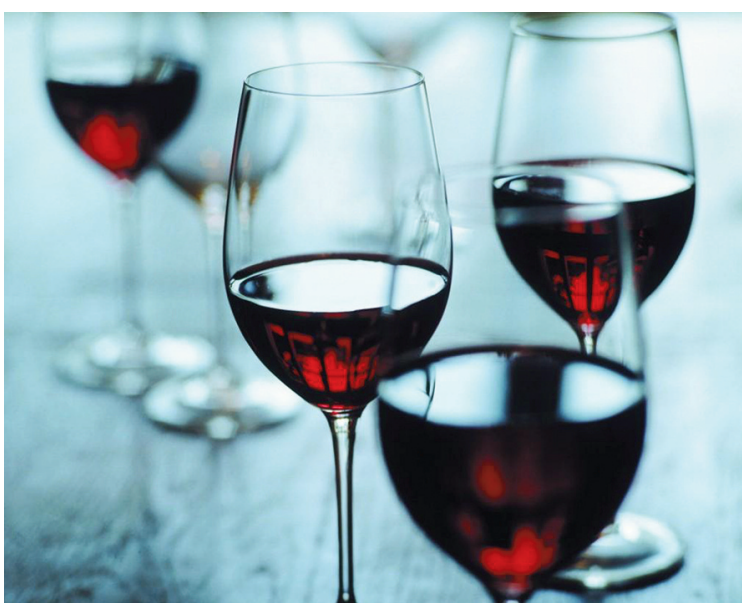

Figure 1. Sicilian wine

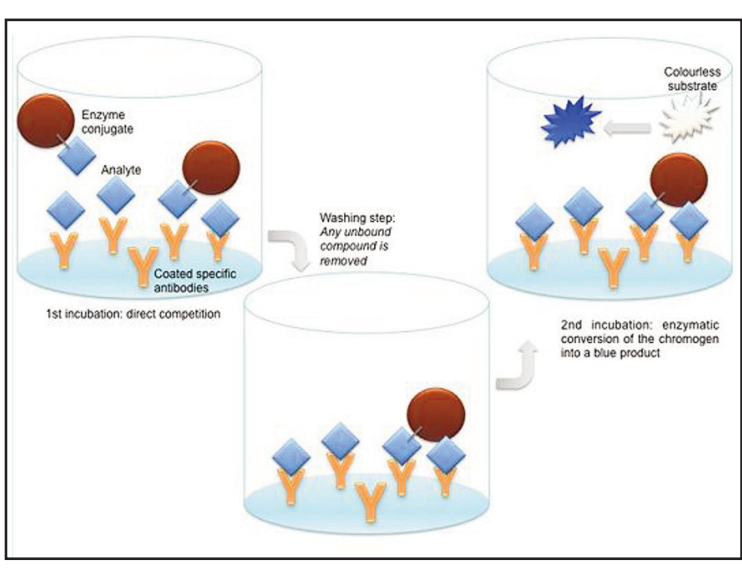

Figure 2. ELISA scheme functioning.

duced in Sicily to obtain a detailed risk assessment of these substances.

\section{MATERIAL AND METHODS}

A total of 470 wine samples based were analised by screening analysis method based on immunoenzymatic assay for the quantitative analysis of ochratoxin. The assay is performed in polystyrene microwells which have been coated with antibodies (IgG) anti - IgG of rabbit. Ocratoxin A standard solution or samples, the enzyme conjugate ochratoxin-HRP and a specific antibody anti-ocrhatoxin were added to the microwells. During the incuba- tion, free ochratoxin-A mulecules and ochratoxin HRP compete for the anti - ochratoxin antibodies binding sites. The anti-ochratoxin antibodies are simultaneously bound to the solid fase. Any unbound enzyme ochratoxin - HRP is then removed in a washing step.

The bound enzyme (HRP) activity is determined by adding a fixed amount of a chromogenic substrate. The enzyme converts the colourless chromogen into a blue product. The addition of the stop reagent leads to a color change from blue to yellow. The absorbance was measured by a microplate reader at $450 \mathrm{~nm}$. The colour development is inversely proportional to the ochratoxin-A concentration in the sample (Figs. 1, 2).

\section{RESULTS}

The results of the present work confirmed the presence of contamination by OTA in only one sample of red wine "Nero D'Avola" from south-oriental Sicily. Specifically in the year 2017 they were examined at the laboratories of the IZS Sicilia in the area of Chemical and Food Technologies 29 samples of officially withdrawn ASP from Sicilian territory. Of these samples 28 are below the detection limit of the method, while only one sample was found to be inadequate to ELISA screening because it exceeded the legal limit established by the Reg. 1881/2006/CEE. The results confirmed good standard practices of Sicilian industry for wine production.

\section{REFERENCES}

Pfohl-Leszkowicz A. \& Manderville A., 2007. Ochratoxin A: an overview on toxicity and carcinogenicity in animals and humans. Molecular Nutrition \& Food Research, 51: 61-99. https://doi.org/10.1002/mnfr. 200600137

IARC, International Agency for Research on Cancer, 1993. Ochratoxin A. In: Some Naturally Occurring Substance, Food Items and Constituents, Heterocyclic Aromatic Amines and Mycotoxins. IARC Monographs on the Evaluation of Carcinogenic Risks to Humans, 56: 489-521.

Majerus P. \& Otteneder H., 1996. Detection and occurrence of ochratoxin $\mathrm{A}$ in wine and grape juice. Deutsche Lebensmittel-Rundschau, 92: 388-390. 